\title{
LAS PIEDRAS LABRADAS 2, 4 Y 5 DE BONAMPAK Y LOS REYES DE XUKALNAH EN EL SIGLO VII
}

\author{
PÉTER BíRÓ \\ Universidad La Trobe, \\ Melbourne, Australia
}

Los textos de Bonampak escritos con jeroglíficos mayas se encuentran en diversos monumentos del sitio: cuatro estelas, cuatro dinteles, cuatro piedras labradas y en el interior de los tres cuartos del Edificio 1 (Mathews, 1980; Arellano, 1998). Otros monumentos de procedencia desconocida mencionan el glifo emblema $A k^{\prime}$ e, relacionado con el sitio, aunque existen varios problemas con esta hipótesis (Beliaev-Safronov, 2004). ${ }^{1}$

Las estelas 1, 2, 3 y 5, los dinteles 1, 2, 3 y los murales del Edificio 1 fueron dedicados durante el gobierno de Yajaw Chan Muwan II (776-796?), mientras que otros monumentos están conectados con gobernantes distintos y sus fechas varían según las reconstrucciones realizadas por diversos autores (Mathews, 1980; Grube-Schele, 1995; Arellano, 1998; Beliaev, 2001; Tovalín, 2002; Wagner, 2004).

En este artículo propongo nuevas interpretaciones sobre las fechas de las piedras labradas 2, 4, y 5, las cuales, aunque no tienen fechas de cuenta larga, presentan varios indicios que permiten ubicarlas hacia el siglo vII. Entre los personajes mencionados en sus inscripciones se encuentra un gobernante de Yaxchilán, antes desconocido, del período del hiato de este sitio (Mathews, 1988; Martin-Grube, 2000), llamado Itsamnaj B'ahlam II.

En la lectura de cada una de las piedras labradas realizaré la transliteración, transcripción y traducción del monumento; proporcionaré asimismo las fechas y los razonamientos de las interpretaciones anteriores, así como los nuevos fechamientos que sugiero, junto con su justificación; finalmente indicaré las implicaciones de esta propuesta. ${ }^{2}$ También incluiré varios dibujos y fotos de los monumentos con el fin de probar que la documentación, en cierto sentido, puede ser la causa de la discordancia en las interpretaciones.

\footnotetext{
${ }^{1}$ En un próximo artículo escribiré sobre la problemática de los glifos emblemas en general y, especialmente, la relación entre el glifo emblema $A k^{\prime} e$ y Bonampak.

${ }^{2}$ En la transliteración y transcripción uso la siguiente forma. En la transliteración los logogramas se escriben en negritas mayúsculas y los silabogramas en negritas minúsculas. El asterisco (*) siempre se antepone al signo de referencia, lo que indica que el signo es reconstruido. La trans-
} 


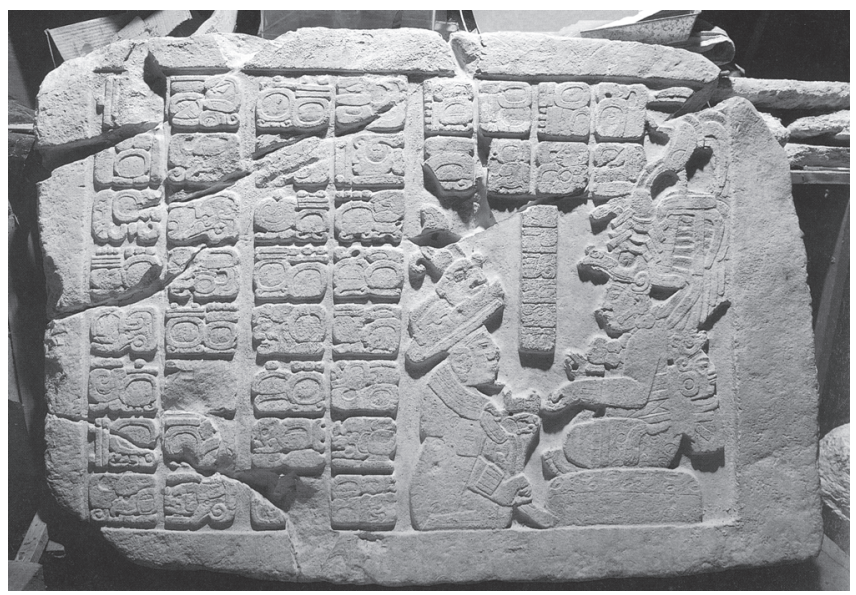

Figura 1. Piedra labrada 4 Arellano, 1998: 272, lámina 6

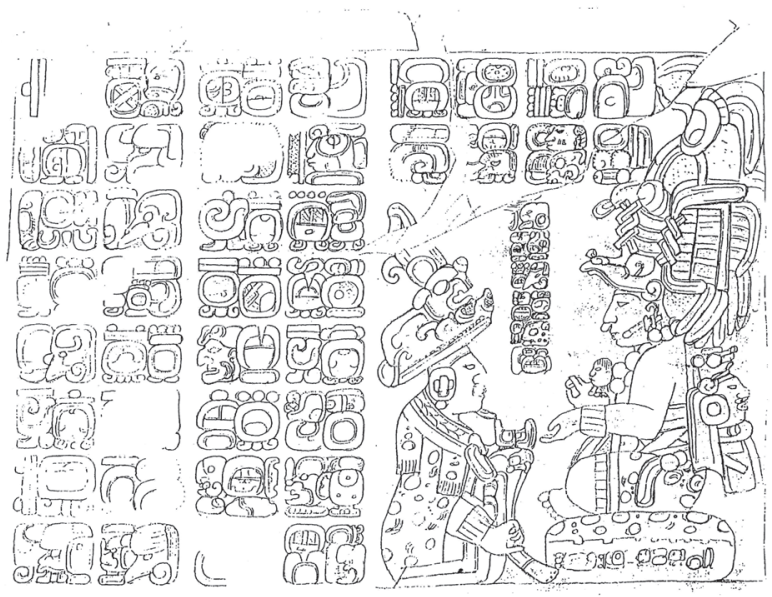

Figura 1a. Piedra labrada 4

Dibujo de Ian Graham ${ }^{3}$

cripción se escribe en cursiva y las reconstrucciones en corchetes [...]. En este artículo uso la transliteración y transcripción en un sentido amplio, es decir, no indico las vocales complejas (Houston, Stuart y Robertson, 1998; Lacadena y Wichmann, 2004), las cuales posiblemente existieron en la lengua de las inscripciones, sin embargo la evidencia no es inequívoca (Mora-Marín, 2003). La traducción está debajo de la transliteración entre comillas “...". En la traducción aplico las ideas de Robert Wald $(2000,2004)$, es decir, asumo que los textos de la época Clásica estaban escritos sin indicar el aspecto, con excepción de algunos verbos en perfectivo (MacLeod, 2004), y porque el discurso relata acontecimientos pasados usaré el pretérito de la lengua castellana.

${ }^{3}$ Sculptured Stone 4 of Bonampak, after field drawing by lan Graham, reproduced courtesy of the Corpus of Maya Hieroglyphic Inscriptions, Peabody Museum, President and Fellows of Harvard College. 


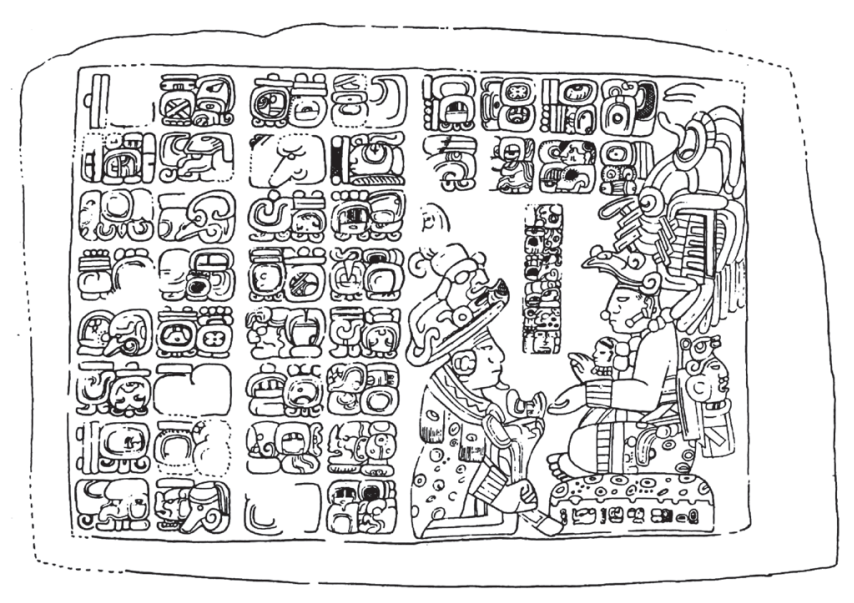

Figura 1b. Piedra labrada 4

Dibujo de Alfonso Arellano (1998: 270, figura 12)

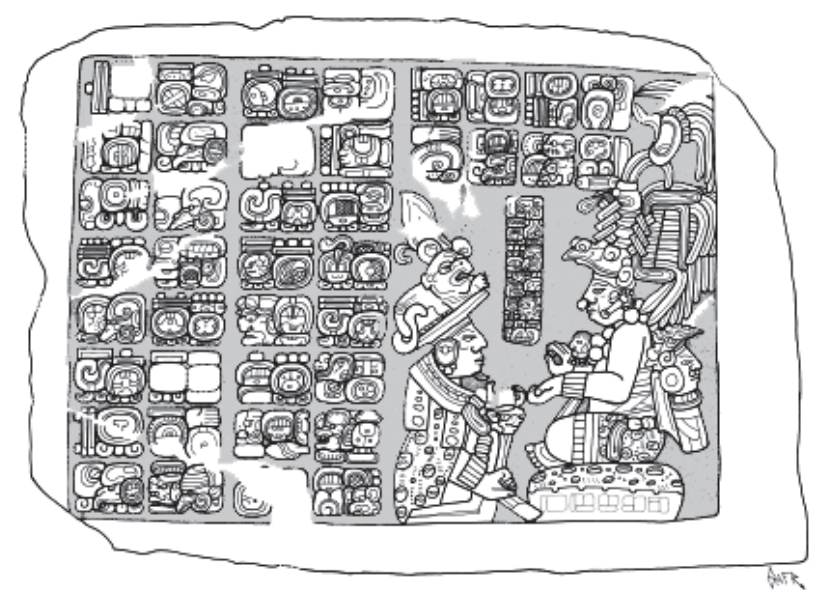

Figura 1c. Piedra labrada 4

Dibujo de Alexandr Safronov

A1 11 * Kab'an

B1 5 Sip CHUM-ji-ya

A2 ta-AJAW-wa-le

B2 ja-ya-MUWAN-CHAN

A3 u-ti-ya
11 kab'an

5 sip chumjiy

ta ajawle[l]

yaja[w] chan muwan

ulh/tiy 
B3 u?-UKUL?

A4 19-he-wa 3-WINIK-?

B4 CHUM-ji-ya-i-ts'a-pa-ja

A5 TUN-ni-li

B5 12 Kib' 4 Yaxk'in

A6 5-he-wa 6-WINIK-ya

B6 5-"HAB'-"ya *i-" u-" ti

A7 12 Ik'

B7 5 Mol k'a-yi

A8 ja-ya-MUWAN-CHAN

B8 ka-xu-NAH-AJAW

C1 6 Muluk $12 \mathrm{Mol}$

D1 CHUM-la-ja

C2 *ta-AJAW

D2 AJ-?-?-li-NAL

C3 1-he-wa 12-WINIK-ya

D3 4-HAB'-ya CHUM-ji-ya

C4 10 Ok 8 Pop

D4 LOK'-ya-tu-CH'EN-na

C5 T'AB'-yi-PA'-CHAN-na

D5 10-he-wa 0-WINIK-ya

C6 1-HAB'-" ya 3-Ajaw

D6 chu-ku-ja-?-HIX?

C7 a-?-CHAN-na-TOK'

D7 xu-ka-la-NAH

C8 * AJAW?

D8 ?-yi tu-CH'EN?

E1 5-*WINIK 9-HAB'-ya

F1 0-K'IN-ni?

E2 k'a-yi-ya

F2 ja-ya-CHAN-na-MUWAN

G1 10 Imix 19 Yax

H1 OCH-B'IH-ja

G2 K'UH-i-IX-xi

H2 K'AN-to-ko-?

I1 u-B'AH

I2 ta-k'a-li?-hu-na ukul?

19 hew 3 winik[jiy]

chumjiy i ts'a/h/paj

tunil

12 kib' 4 yaxk'in

5 hew 6 winikljily

5 hab'[jily i u/h]ti

$12 i k^{\prime}$

5 mol k'a'y

yaja/w] chan muwan

xuka[l]nah ajaw

6 muluk $12 \mathrm{~mol}$

chumlaj

ta ajaw[lel]

aj ?-Vl nal

1 hew 12 winikljily

4 hab'[jily chumjiy

10 ok 8 pop

lok'loyily tu ch'en

t'ab'ay pa'chan

10 hew 0 winik[jily

1 hab'[jiy] 3 ajaw

chu/h/kaj ?-hix?

$a$-? chan tok'

Xukalnah

ajaw?

?-iy tu ch'en?

5 winik[jiy] 9 hab'[jily

0 k'in

k'a'yiy

yaja/w] chan muwan

10 imix 19 yax

ochbihaj

k'uh/ul] ix

k'an tok?

u b'ah

ta k'al? ${ }^{4}$ hun

${ }^{4}$ Es difícil interpretar esta parte de la inscripción porque los componentes no se ven bien en ninguna de las fotos en mi poder. No obstante, la expresión u b'ah normalmente se antepone al nombre de un personaje, o con la preposición ta/ti ante la acción que normalmente se representa en la escena. La interpretación: $u$ b'ah ta k'alhun o 'su imagen durante la coronación' es confirmada por la imagen en el monumento. 


\author{
I3 3-WINIK-HAB'-AJAW \\ I4 UH?-Xu?-CHAN? ? ${ }^{5}$-na? \\ I5 ITSAMNAJ-B'ALAM \\ I6 K'UH-PA'-CHAN-AJAW
}

3 winikhab' ajaw
Uhux?-chan ?-an
itsamnaj b'alh/lam
k'uh[ul] pa'chan ajaw

\section{Traducción libre}

“En 11 Kab’an 5 Sip Yajaw Chan Muwan se sentó en el reino que ocurrió en Ukul?, 19 días y 3 veintenas después de su entronización, en 12 Kib’ 4 Yaxk’in, él [Yajaw Chan Muwan] erigió una estela, 5 días, 6 veintenas y 5 períodos de 360 días después, en 12 lk' 5 Mol, Yajaw Chan Muwan, el gobernante de Xukalnah, murió. En 6 Muluk 12 Mol, Aj? Nal se sentó en el reino, 1 día, 12 veintenas y 4 períodos de 360 días después de su entronización, en 10 Ok 8 Pop, salió de su lugar y se exilió? para Pa'chan, 10 días, 0 veintenas y 1 período de 360 días después, en 3 Ajaw [13 Pop], Aj Chan? Tok', el gobernante? de Xukalnah, fue capturado, y él [Aj? Nal] regresó? para su lugar. 0 día, 5 veintenas y 9 períodos de 360 días después de la muerte de Yajaw Chan Muwan, en la noche de 10 Imix 19 Yax, la Señora Sagrada K’an Tok entró en la ruta [muerte].

Su imagen durante la coronación [por] el 3 Winikhab' Señor, Uhux? Chan ?an, Itsamnaj B'ahlam, Sagrado Gobernante de Pa'chan”.

La piedra labrada 4 fue encontrada en 1981 durante las excavaciones arqueológicas dirigidas por Mario Pérez Campa (INAH), en la escalera principal del Edificio 8 (Miller, s. f.; Wagner, 2004). Es muy probable que estuviera originalmente colocada sobre la pared del Edificio 8, por lo que sería mejor designarla como 'panel' en vez de 'piedra labrada', aunque se respetará la nominación dada para evitar confusiones.

El monumento tiene 51 bloques de glifos, de los cuales 40 encuadran dos personajes sentados, seis bloques se encuentran entre ellos, y otros cinco, que nunca fueron adecuadamente documentados, se localizan en el trono cubierto con una piel de jaguar (figura 1c).

Las ruedas calendáricas en el texto son las siguientes:
11 * Kab'an
5 Sip
12 Kib'
4 Yaxk'in
$+5.6 .5$

\footnotetext{
${ }^{5}$ Aunque este glifo se compone de dos logogramas CHAK (T109) y KAB' (T526), es muy probable que sea el nombre de un ave rapaz y los logogramas forman un signo compuesto con una lectura diferente que se termina en -n. Su substitución con un logograma que representa la cabeza de un ave en las inscripciones de Yaxchilán, Palenque y en varias cerámicas, es la base de esta hipótesis por primera vez planteada por Nikolai Grube (s. f.).
} 


$\begin{array}{ll}12 \mathrm{Ik}^{\prime} & 5 \mathrm{Mol} \\ & \\ 6 \text { Muluk } & 12 \mathrm{Mol} \\ & +4.12 .1 \\ 10 \mathrm{Ok} & 8 \mathrm{Pop} \\ & +1.0 .10 \\ 3 \mathrm{Ajaw}^{*} & 13 \text { * Pop } \\ {\left[12 \mathrm{Ik}^{\prime}\right.} & 5 \mathrm{Mol}] \\ & +9.5 .0 \\ 10 \mathrm{Imix} & 19 \mathrm{Yax}\end{array}$

Existen varias posibilidades de cuentas largas para el lapso comprendido en este grupo de ruedas calendáricas:

9.5.14.0.17 11* Kab’an 5 Sip-9.6.8.12.1 10 Imix 19 Yax (548-562)

0

9.8.6.13.17 11\% Kab’an 5 Sip -9.9.1.7.1 10 Imix 19 Yax (600-614)

o

9.10.19.8.17 11* Kab’an 5 Sip -9.11.4.2.1 10 Imix 19 Yax (652-666)

o

9.13.12.3.17 11* Kab'an 5 Sip -9.14.6.15.1 10 Imix 19 Yax (704-718)

o

9.16.4.16.17 11* Kab’an 5 Sip -9.16.19.10.1 10 Imix 19 Yax (756-770) o

9.18.17.11.17 11* Kab’an 5 Sip -9.19.12.5.1 10 Imix 19 Yax (808-822)

Se podría decir, por el estilo, que las dos últimas posibilidades están descartadas. Sin embargo, la mejor ayuda para restringir las fechas es la mención en el texto de un Itsamnaj B'ahlam K'uhul Pa'chan Ajaw, o sea, 'Sagrado Señor de Pa'chan' (Yaxchilán, en I1-I6), quien era el gobernante de Yaxchilán cuando se dedicó el monumento. Así, las únicas cuentas largas que cumplen esta condición son las siguientes:

9.8.6.13.17 11* Kab'an 5 Sip-9.9.1.7.1 10 Imix 19 Yax (600-614)

0

9.13.12.3.17 11* Kab’an 5 Sip -9.14.6.15.1 10 Imix 19 Yax (704-718)

0

9.16.4.16.17 11* Kab’an 5 Sip -9.16.19.10.1 10 Imix 19 Yax (752-770)

o

9.18.17.11.17 11* Kab’an 5 Sip -9.19.12.5.1 10 Imix 19 Yax (808-822)

En el primer y último caso no se conocen los nombres de los gobernantes de Yaxchilán. Durante el período de 9.16.4.16.17 hasta 9.16.19.10.1 se sabe que el 
gobernante de Bonampak se llamaba Aj Sak Telech (Miller-Martin, 2004), que difiere de los gobernantes mencionados en la piedra labrada 4. En el caso del 9.18.17.11.17-9.19.12.5.1 infiero que se trata de una fecha muy tardía para la región porque ni en Yaxchilán ni en Bonampak hay inscripciones para ese momento (Mathews, 1988; Martin-Grube, 2000) o para 822.

Autores anteriores como Grube y Schele (1995: 154), y Arellano (1998: 264) prefirieron la cuenta larga 9.13.12.3.17-9.14.6.15.1 (704-714), asumiendo que el gobernante mencionado era Itsamnaj B'ahlam II (681-742). Otros, como Wagner (2004) y Miller (s. f.) sugieren después que el estilo era más temprano y proponen la cuenta larga 9.8.6.13.17-9.9.1.7.1 (600-614), con ello se implica que Itsamnaj B’ahlam era un gobernante antes desconocido para Yaxchilán.

¿Existe una manera de decidir entre estas dos cuentas largas? En mi opinión existen varias líneas de evidencia que muestran que la fecha más temprana es la correcta, y que Itsamnaj B'ahlam fue un gobernante de Yaxchilán durante su hiato, ocurrido entre 564-629.

Una clave para esta interpretación es la lectura de los glifos en I1-I2. En la escena se aprecian dos personas, por lo que sería razonable sugerir que ambas están mencionadas, sin embargo, se trata de un solo nombre y los títulos están relacionados con el gobernante de Yaxchilán. Desafortunadamente, las fotos y los dibujos en mi poder no son claros, siendo imposible leer todos los signos del nombre, aunque es factible presentar un análisis de los principales componentes (figura 2).

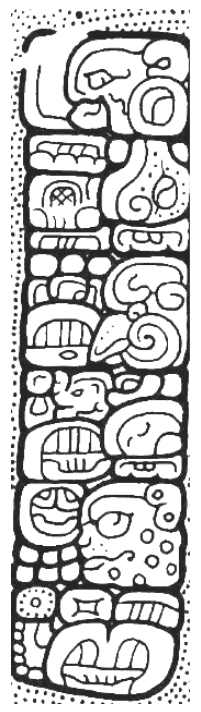

Figura 2. El NOMBRE DE UH?-Xu?-CHAN ?-NA ITSAMNAJ-B'ALAM en piedra labrada 4 Dibujo de Alexandr Safronov 


\section{a. u-B'AH ta-k'a-li?-hu-na UX-WINIK-HAB'-AJAW UH-xu?-CHAN-'CH'AK-KAB' '-na ITSAMNAJ-B'ALAM K'UH-PA'CHAN-AJAW}

b. u b'ah ta k'al?hun ux winikhab' ajaw uhux? chan ?-an itsamnaj b'a/h/lam k'uh[ul] pa'chan ajaw

c. "su imagen durante la coronación [por] el 3 Winikhab' Señor, Vx? Chan ?-an, Itsamnaj B’ahlam, Sagrado Señor de Yaxchilán”

Los componentes, salvo el Itsamnaj B'ahlam, son diferentes del nombre del gobernante que ocupó ese cargo entre 681-742 en Yaxchilán. Sin embargo, esto no es suficiente para afirmar que hay una diferencia entre los dos personajes porque, como se sabe, muchos gobernantes mayas tenían nombres muy largos que no eran usados en cada inscripción (Grube, 2001; Colas, 2003).

También se conoce que cuando este gobernante estaba en su tercer winikhab' (período de 20 tun), se dedicó el monumento, lo que tuvo que ocurrir cercanamente a la última fecha del texto (614 o 718); él habría tenido entre 40 y 60 años de edad, lo que lo aparta de Itsamnaj B'ahlam II, quien se supone nació entre 643 - 647 y se entronizó en 681. Teniendo en cuenta su fecha de entronización, Itsamnaj B'ahlam II habría estado en su segundo winikhab' (de gobierno) en 718, y ya habría pasado su cuarto winikhab' de vida.

De acuerdo con los argumentos anteriores, es muy improbable que el personaje mencionado en la piedra labrada 4 de Bonampak sea el mismo personaje que gobernaba Yaxchilán entre 681-742. Sugiero entonces que los personajes mencionados en el texto son: un gobernante de Yaxchilán desconocido antes, dos gobernantes de Xukalnah (uno mencionado explícitamente, el segundo es una implicación), un cautivo del mismo sitio, una mujer con el nombre de Ix K'an Tok ? y el escultor del monumento.

El nombre del primer rey de Xukalnah se lee según el orden de los glifos ja-yaCHAN-MUWAN o, jay chan muwan, quien gobernaba entre 600 y 605. Pero, justamente en este período, el dintel 4 de la Estructura 6 de Bonampak se fecha estilísticamente en 9.8.9.15.11 (603) y conmemora una victoria contra alguien de Lakam Ha' (Grube, 1996). El gobernante mencionado en este texto se llama ? ${ }^{6}$-si ?-na ya-ja-CHAN-na MUWAN, o sea, ?-s ?-an yaj chan muwan (figura 3, incisos a y b).

Una de las características de la escritura maya es la posibilidad de variar la posición de los signos, como ocurre aquí con los dos silabogramas ya y ja. Solamente en el texto de la piedra labrada 4 se aprecian dos diferentes maneras de

\footnotetext{
${ }^{6}$ La primera sílaba del nombre de este gobernante y su homónimo del Clásico Tardío, en ambos casos, se compone de TI' (la variante de cabeza sin número de Thompson y T128) boca, K'IK'? (T628) sangre y el silabograma si. A veces T629 se encuentra en la boca de la variante de cabeza del glifo T128, y sangre T128 está antepuesto (en K5465). En el caso de la combinación de Tl' y otro glifo, la lectura del glifo compuesto difiere de sus componentes individuales: TI' + WAJ $=$ WE' $\sim$ comer, $\mathrm{Tl}^{\prime}+\mathrm{HA}^{\prime}=\mathrm{UK}^{\prime} \sim$ beber, $\mathrm{Tl}^{\prime}+\mathrm{MO}^{\prime}=\mathrm{NUN} \sim$ diluvio? Entonces, es probable que la combinación Tl'+K'IK'?-si indique un morfema que termina en -s. (véase sobre este glifo el comentario de Nikolai Grube para el vaso K8777 en el sitio internet de famsi).
} 


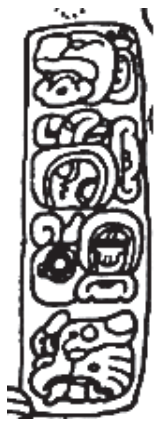

a)

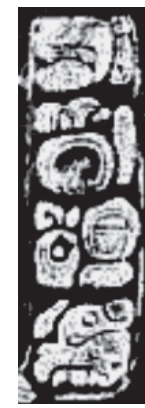

b)

Figura 3. Nombre de ?-Na ?-Si Yajaw Chan Muwan

a) dintel 4 . Dibujo de Linda Schele; ${ }^{7}$

b) dintel 4. Calca hecha por Merle Green Robertson

escribir Xukalnah: la primera, ka-xu-NAH-AJAW y, la segunda, xu-ka-la-NAH (figura 4 incisos a y b). En el primero caso no representa el fonema 'l' porque se sabe que los escribanos a veces no escribían la primera consonante en un grupo consonántico, en este caso 'In' (Lacadena, 1995; Zender, 1999; Bíró, 2004).

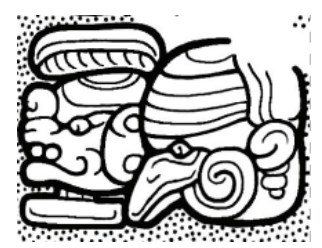

a)

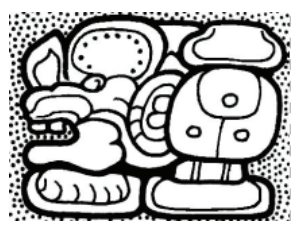

b)

FIGURA 4. a) KA-XU-NAH-AJAW, piedra labrada 4, B: 8. Dibujo de Alexandr Safronov b) XU-KA-LA-NAH, piedra labrada 4, D: 7. Dibujo de Alexandr Safronov

Conocer el nombre del último gobernante de Bonampak - Yajaw Chan Muwan II- resulta útil para reconstruir los nombres de los monumentos como Yaja[w] Chan Muwan, donde el fonema 'w' no fue representado porque era la primera consonante en el grupo consonántico 'wch'.

${ }^{7}$ SD6007 Lintel from Structure 6, Bonampak. Drawing by Linda Schele, (C) David Schele, courtesy Foundation for the Advancement of Mesoamerican Studies, Inc., www.famsi.org. 
Según mi razonamiento, el dintel 4 y la piedra labrada 4 mencionan al mismo gobernante de Xukalnah, ?-s ?-an Yajaw Chan Muwan, quien habría gobernado entre 600 y 605. El único monumento durante ese momento es el dintel 4 de Bonampak, pero pienso que la piedra labrada 2 también fue dedicada por él.

Antes de entrar en la discusión considero importante presentar si se sabe algo más sobre Itsamnaj B’ahlam II (desde aquí el gobernante de Yaxchilán mencionado sobre la piedra labrada 4).

Desafortunadamente, entre 537 (9.5.2.10.6) y 681 (9.12.8.14.1) no hay monumentos contemporáneos en Yaxchilán, salvo la estela 2 que muy probablemente es del 613 (9.9.0.0.0), pero está tan erosionada que es imposible decir cuál es el nombre del protagonista (Mathews, 1988; Martin-Grube, 2000). No obstante, hay varias fechas conocidas de este hiato — 564, 629, 636, 647, 649, 659 y 669conectadas con dos gobernantes, ?-B'ahlam (564) y Yaxun B'ahlam III (629-681?). Además, el gobernante 10, K'inich Tatb'u Jolom II estuvo en el poder al menos hasta 537 (9.5.2.10.6). Werner Nahm ha argumentado que hubo otros dos sucesores mencionados en la escalera jeroglífica I de Yaxchilán, y ambos tenían un 'nudo' en su nombre, lo que sugiere que podrían haber compartido el mismo apelativo que ?-B'ahlam (Nahm, 1997).

Se sabe que Yaxun B'ahlam III era el gobernante $15^{\circ}$ del sitio (Martin-Grube, 2000), teniendo la siguiente lista de gobernantes:

$\begin{array}{ll}\text { LUGAR } & \text { NomBRE } \\ 10^{\circ} & \text { K'inich Tatb'u Jolom }(>526-537<) \\ 11^{\circ} & \text { ?-B'ahlam } \\ 12^{\circ} & \text { ?-B'ahlam }(>564<) \\ 13^{\circ} & \\ 14^{\circ} & \text { Itsamnaj B'ahlam III }(>614<) \\ 15^{\circ} & \text { Yaxun B'ahlam III }(629-681 ?)\end{array}$

Es imposible decir con absoluta certeza si el Itsamnaj B'ahlam de la piedra labrada 4 era el $13^{\circ}$ o $14^{\circ}$ gobernante. Sin embargo, de acuerdo con el patrón del Clásico Tardío es probable que fuera el predecesor directo de Yaxun B’ahlam III. Su título de 3 winikhab' sugiere que Itsamnaj B'ahlam II nació alrededor de 554. En tal caso el hijo de ?-B'ahlam podría ser el $12^{\circ}$ gobernante.

La relación entre Xukalnah y Yaxchilán se remonta al Clásico Temprano, y además la dinastía de Xukalnah ya está mencionada en las inscripciones más tempranas de la región. El primer gobernante de Xukalnah está mencionado en la estela 1 de Ojos de Agua. En esta escultura ?-Pat, el rey de Ojos de Agua, conmemora su entronización en 573 y se declara el séptimo descendiente ( $u$ wuk ts'akb'ul) de un yajawte', el cual, a su vez, era hijo de Aj Yax Punim, el gobernante de Xukalnah (figura 5). Según esta información, Aj Yax Punim vivió al menos ocho generaciones antes de 573, si cuento 20 años por cada generación, él pudo haber vivido a comienzos del siglo v (Beliaev-Safronov, 2004). 
Es muy probable que Aj Yax Punim fuera el fundador de una dinastía proveniente de una rama de la familia real de Xukalnah. Cabe recordar que el primer contacto entre Yaxchilán y Xukalnah, al menos registrado en las inscripciones, es la captura de ? Xute'l K'an Tok Luk Xukalnah Ajaw por ?-B'ahlam, en 564, mencionado en el escalón 1 de la escalera jeroglífica III de Yaxchilán y en la estela 18 del mismo sitio (figura 6 incisos a y b).

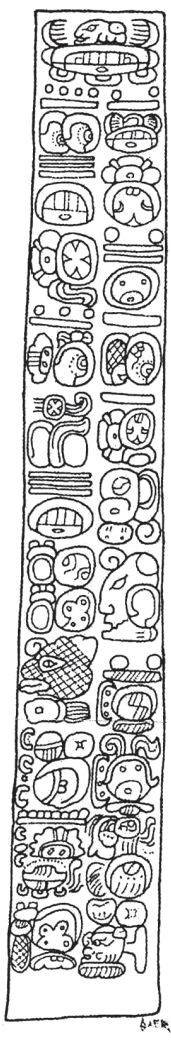

Figura 5. Estela 1 de OJos de Agua Dibujo de Alexandr Safronov

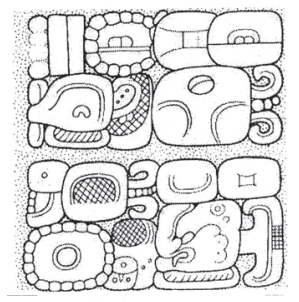

a)

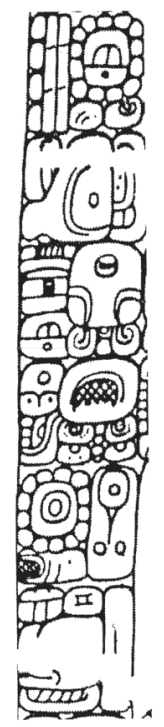

b)

Figura 6. El nombre de Aj Popol Chay A[J] Payal Mo'l Xukalnah Ajaw a) escalera jeroglífica 3 de Yaxchilán Dibujo de Ian Graham (1982: 166) b) estela 18 de Yaxchilán Dibujo de Ian Graham (Tate 1992: 246, figura 145b) 
La piedra labrada 4 de Bonampak presenta de nuevo un lazo estrecho entre Xukalnah y Yaxchilán. Yajaw Chan Muwan I de Xukalnah fue entronizado en 600 en Ukul, y murió en 605. Su sucesor fue Aj ? Nal, quien huyó del sitio y fue a Yaxchilán. No se menciona la causa de su exilio, aunque de la narrativa se infiere que el cautivo Aj ? Chan Tok' estuvo involucrado. Su captura en 611 está ligada directamente con un verbo que puede indicar el retorno a la ciudad (Beliaev, 2001). La estancia de Aj ? Nal en Yaxchilán duró un año, lo que puede indicar la buena relación con su gobernante, posiblemente ya como Itsamnaj B'ahlam II.

La representación de Itsamnaj B’ahlam II no sólo es un indicador de su importancia en este evento particular, sino también como el señor supremo de $\mathrm{Aj}$ ? Nal. El texto termina con la muerte de una cierta Ix K'an Tok ? (Wagner, 2003, 2004), la cual guarda relación directa con la muerte de Yajaw Chan Muwan I. ¿Cuál es la relación de esta señora con los personajes mencionados en el texto, especialmente Yajaw Chan Muwan I? Opino que la respuesta se encuentra en el texto de la piedra labrada 2 de Bonampak que examinaré a continuación.

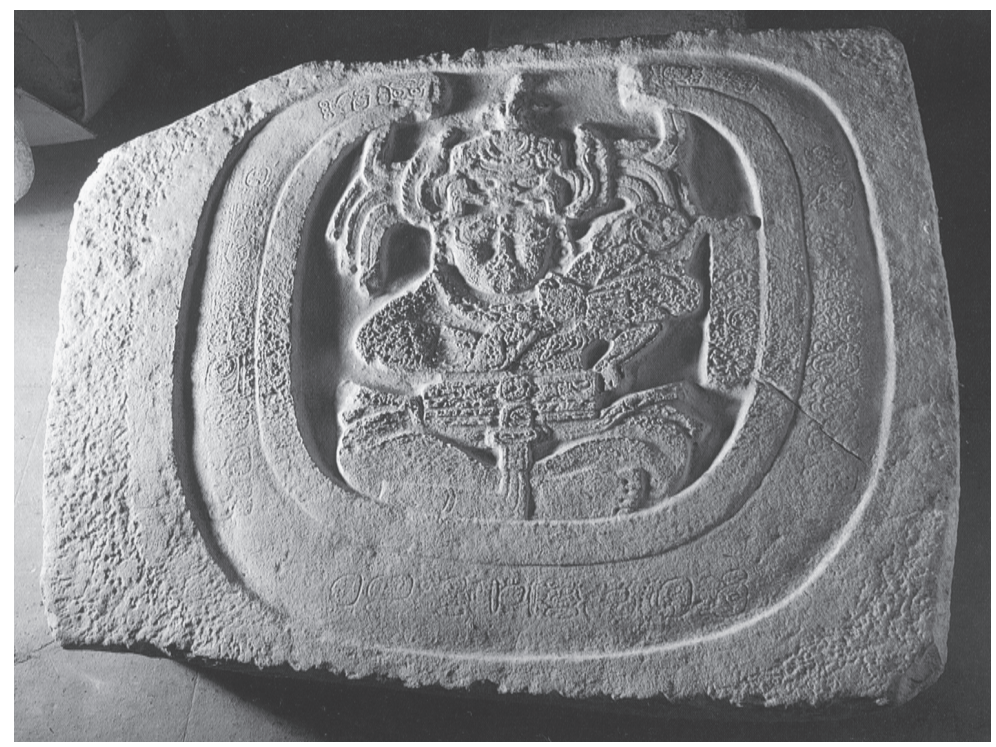

Figura 7. PiedRa labrada 2 Arellano, 1998: 274, lámina 7 
Figura 7a. Piedra labrada 2 Dibujo de Linda Schele ${ }^{8}$
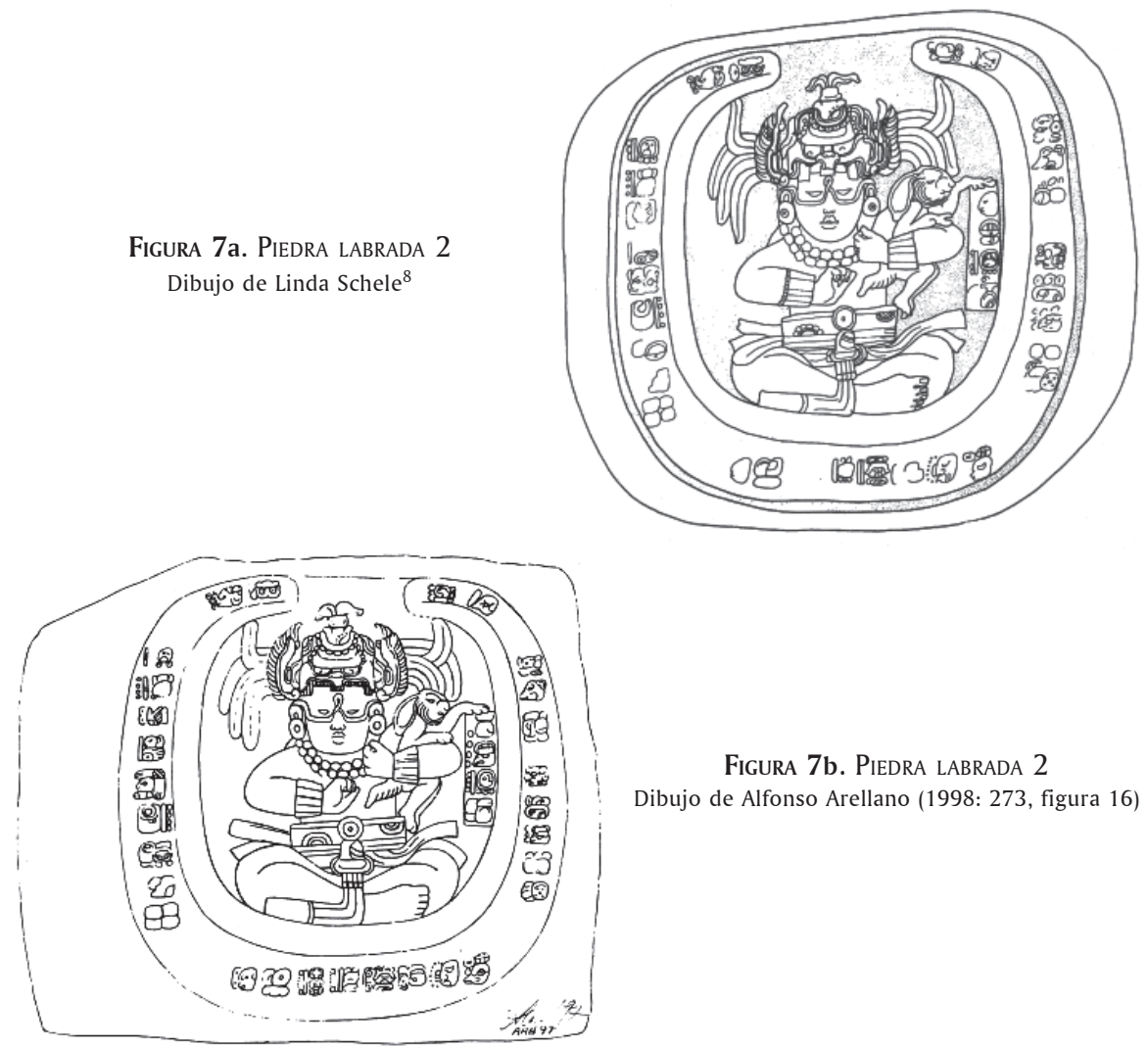

Figura 7b. Piedra labrada 2

Dibujo de Alfonso Arellano (1998: 273, figura 16)

Figura 7c. Piedra labrada 2

Calca hecha por Merle Greene Robertson

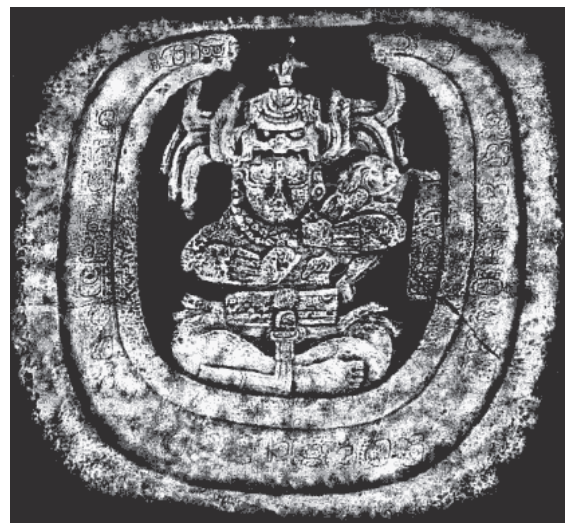

${ }^{8}$ SD6008 Stone panel, carve, Bonampak. Drawing by Linda Schele, (c) David Schele, courtesy Foundation for the Advancement of Mesoamerican Studies, Inc. www.famsi.org. 


\begin{tabular}{|c|c|}
\hline A1 5-ta-Ajaw & 5 ta ajaw \\
\hline A2 9? & $9 ?$ \\
\hline A3 5 HUL-"*i-"'ya & 5 huliy \\
\hline A4 5?-K'AL-?-ja & 5? k'al-?aj \\
\hline A5 B'ALAM-K'UH-CHAPAT & b'a/hllam k'uh chapat \\
\hline A6 B'ALUN-KAL? & b'alun kal? \\
\hline A7 *3-Wayeb’ & 3 wayeb' \\
\hline A8 T’AB'-yi & t'ab'ay \\
\hline A9? & $?$ \\
\hline B1 *0?-"6?-"WINIK-? & 0 [hew] 6 winik \\
\hline B2 3-HAB'? & 3 hab'? \\
\hline B3 9 Ajaw? & 9 ajaw? \\
\hline B4? & $?$ \\
\hline C1 xu-ka-la-NAH & Xukalnah \\
\hline C2 AJAW & ajaw \\
\hline C3 ? & $?$ \\
\hline C4? & $?$ \\
\hline C5 'TI'-K'IK' ‘ & ? \\
\hline C6 'СНAK-KAB’ ‘ & ? \\
\hline C7 ya-?-MUWAN & ya[jaw chan] muwan \\
\hline C8? & ? \\
\hline C9 ?-b’i & $? b^{\prime}$ \\
\hline D1 IX? & $i x ?$ \\
\hline D2 sa-ja-la & sajal \\
\hline D3 IX-K'AN-to-ko-? & ix k'an tok? \\
\hline D4 ? & $?$ \\
\hline D5 u-MIHIN?-li & umihinil? \\
\hline D6? & $?$ \\
\hline D7 K'UH & k'uh[ul] \\
\hline D8 a-k'e-AJAW & ak'e ajaw \\
\hline E1 yu-xu-lu & yuxul \\
\hline E2 na-na-EK' & nan ek' \\
\hline E3 AJ-K'UH?-HUN-na? & aj k'uhun? \\
\hline E4 ?-AJAW & ? ajaw \\
\hline
\end{tabular}

Traducción libre

"En 5 Ajaw, cuando el señor de la noche es ?, la luna tenía cinco días de haber nacido, se completó la ? luna, B'ahlam K'uh Chapat [era su nombre] y duraría 29 días, en 3 Wayeb', se dedicó, ?, "0 días, "6 veintenas y 3 períodos de 360 días después, en 9 *Ajaw, el gobernante de Xukalnah ?, ?, ? ?-s ?-an Yajaw Chan Muwan, ?, 
[su madre?] es Ix Sajal, ella de K'an Tok ?, su padre es ?, sagrado gobernante de Ak'e. La escultura de Nan? Ek', Aj K'uhun? y ? señor".

La piedra labrada 2 fue encontrada cerca de la estela 3, pero seguramente no fue su ubicación original (Ruppert et al., 1955). La piedra representa a un hombre sentado "dentro de la luna" con una máscara - lo que representa una anteojera del Sol Jaguar del Inframundo (comunicación personal con Maricela Ayala, 2005) - y un conejo en su mano. Es probable que el personaje personifique la diosa de la Luna; símbolo de fertilidad y capacidad de renacer, entre otros. Aunque la personificación de un hombre en una diosa parezca extraña, hay ejemplos de la región de Palenque donde personajes masculinos aparecen ataviados por la falda de esta diosa (Miller-Martin, 2004: 96-97).

El texto originalmente tenía 34 bloques de glifos, de los cuales muchos están muy deteriorados, lo que dificulta identificar la fecha y los nombres. No obstante, la foto publicada por Alfonso Arellano (1998: 274) y la calca realizada por Merle Greene Robertson ayudan a aclarar al menos una de las fechas y las relaciones entre las cuatro personas mencionadas en el texto. Autores anteriores han propuesto varias lecturas para las fechas. Proskouriakoff señaló que la piedra labrada 2 pertenece, estilísticamente, al comienzo del Clásico Tardío (en Ruppert et al., 1955: 30-31).

Peter Mathews (1980) propuso que la primera fecha se componía de 5 Ajaw y G1, 5D, 5C, X2, A9 de la Serie Lunar y un final de período, o 0 Wayeb'. Sin embargo, él mismo observó que 5Cy X2 no podían estar juntos. Además, Mathews sugirió que había un número de distancia 3.0.2 o 3.2.0 en B1-B2 y un 9 Ajaw en B3. Un problema con este fechamiento es el glifo que él interpreta como G1 y que parece ser G6, en la misma forma que aparece en la estela 6 de Yaxchilán (figura 8). Por otro lado, 0 Wayeb' no corresponde si el día es Ajaw, ya que éste requeriría un número $3,8,13$ o 18 .

Tomando en cuenta esos problemas, Arellano (1998: 267-268) propuso una lectura de 5 K'imi 9 Kayab' con G1, 15D, 5C, B, A9 y la segunda fecha, después de un número de distancia de 3.0.3, sería 9 Muluk 17 Pax. La fecha en cuenta larga propuesta es 9.14.2.2.6-9.14.5.2.9.

Un examen detenido de la foto de Arellano (figura $7 \mathrm{~b}$ ) señala que la primera fecha es 5 Ajaw escrita como 5 ta ajaw, o sea, "en 5 Ajaw". Entonces, el siguiente glifo no puede ser el hab' porque lleva un coeficiente 9, pero se ve exactamente como G6. Sin duda alguna, el coeficiente del glifo D es 5, mientras el coeficiente del glifo $\mathrm{C}$ es $5+$. Los siguientes glifos son $\mathrm{X} 2$ y A9, en tanto el mes de hab' se compone de una cabeza y WAY-HAB' o Wayeb'.

Por eso, a diferencia de las lecturas planteadas, mi lectura de la primera fecha es 5 Ajaw, G6, 5D, 5+C, X2, A9 ? Wayeb'. Ahora bien, ¿cuáles son la cuenta larga y el coeficiente del $h a b$ '? Seguramente el monumento corresponde al b'aktun 9 y el coeficiente del winal puede ser 3/12 y 0 para el k'in, lo que permite la concordancia del día Ajaw con G6. Por ello, el coeficiente del Wayeb' tiene que ser 3, 8, 13 o 18 . 


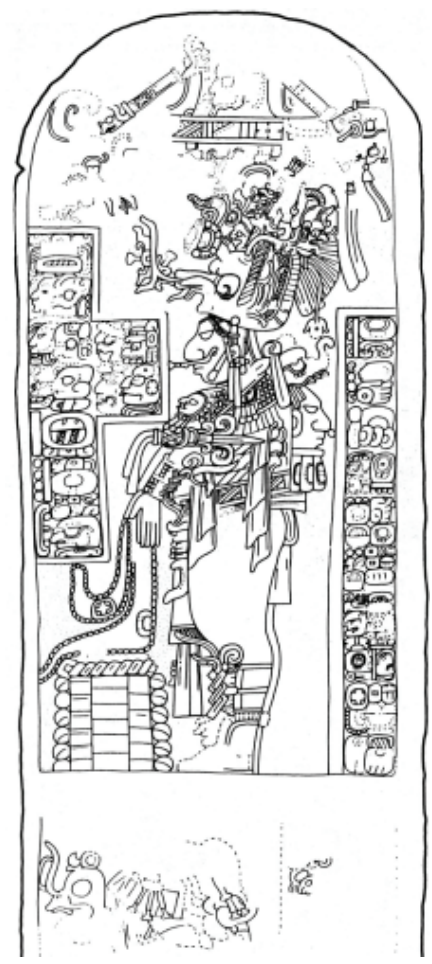

Figura 8. Estela 6 de Yaxchilán Dibujo de Carolyne Tate (1992: 193, figura 88a)

La clave de mi interpretación para asociar esta rueda calendárica con una cuenta larga es la lectura de los glifos en C5-C7. En la calca de Merle Green Robertson, en $\mathrm{C} 5$ se ve claramente una cabeza con algo en la boca. C6 se lee “CH"AK-KAB' “, mientras en $\mathrm{C} 7$ se encuentra un silabograma ya con el logograma MUWAN. Los únicos personajes que tienen en su nombre al menos todos estos componentes son "TI"-K'IK'? “-si “CH”AK-KAB' “-na ya-ja-CHAN-na MUWAN del dintel 4 de Bonampak y de la piedra labrada 4, y su homónimo Yajaw Chan Muwan II del siglo viII. ${ }^{9}$ Se observa que el texto habla sobre su padre, un gobernante de Ak'e, mientras que Ix Sajal Ix K'an Tok ? son los títulos de su madre, teniendo en cuenta la sintaxis común de los textos del período Clásico.

Asimismo, la muerte de un Ix K'an Tok ? esta mencionada en la piedra labrada 4 , y la fecha de su fallecimiento está directamente relacionada con la muerte de Yajaw Chan Muwan I. Por esta línea de evidencia, propongo que la piedra la-

\footnotetext{
${ }^{9}$ Sobre los componentes de este nombre véanse las notas 4 y 5 .
} 
brada 2 es un monumento que menciona a Yajaw Chan Muwan I, su madre Ix Sajal Ix K'an Tok ? y a su padre ?, el gobernante de $A k^{\prime} e$. En los casos de la piedra labrada 2 y 5 , el título Ix K'an Tok ? lleva un sufijo que no se ve muy claro. No obstante, en el escalón de la escalera jeroglífica III de Yaxchilán, en B2b-B3 se encuentra el nombre del cautivo 'Etznab'-Xutel Kan Tok Luk, quien fue un ajaw de Xukalnah. Aunque no lleva AJ, es el afijo agentivo en la lengua de las inscripciones clásicas, es muy probable que se trate de un topónimo, o título, llevado por personas que tienen lazos con la entidad política de Xukalnah. Así, es factible que la madre de Yajaw Chan Muwan I fuera originaria del mismo lugar, o tuviera el mismo título, y el sufijo borrado en ambos casos se leería luk.

¿Pero de qué se trata el texto? Se puede observar, después de la primera fecha, el verbo transitivo derivado, T'AB' 'acicalar, ungir' y el nombre del objeto erosionado completamente (A8-A9), mientras que pasando de la segunda fecha resulta que el actor es un gobernante de Xukalnah.

Es bastante improbable que el título del gobernante fuera seguido por el nombre de Yajaw Chan Muwan I, y en C3-C4 se encontrara otra fecha o, mejor, otro verbo. Sin embargo, el personaje más importante es Yajaw Chan Muwan I, quien conmemora su línea familiar. Por ello creo que la piedra labrada 2 puede fecharse entre 9.8.6.13.17 y 9.8.12.2.2; fechas de la entronización y muerte de Yajaw Chan Muwan I.

La única cuenta larga que cumple con los requisitos de 5 Ajaw, G6 y ? Wayeb' es 9.8.8.12.0 5 Ajaw 3 Wayeb' (8 de marzo del 602); es posible que la cabeza frente del hab' Wayeb' sea la del numeral 3. En cambio, la segunda fecha es más difícil de descifrar. Aceptando la propuesta de Peter Mathews (comunicación personal, 2005), tenemos que si el día es 9 Ajaw (Mathews, 1980) entonces el único fin de período antes de la muerte de Yajaw Chan Muwan I sería 9.8.12.0.0 9 Ajaw 3 Xul, que es exactamente 0.6.3 más que de 9.8.8.12.0 5 Ajaw 3 Wayeb'. Según esta reconstrucción las fechas de la piedra labrada 2 son las siguientes:

$$
\begin{aligned}
& \text { 9.8.8.12.0 } 5 \text { Ajaw *3 Wayeb' } \\
& + \text { 3.*6.0 } \\
& \begin{array}{l}
\text { 9.8.12.0.0 9 *Ajaw *3 Xul }
\end{array}
\end{aligned}
$$

La posición del monumento frente a la estela 3 de Yajaw Chan Muwan II, quizá sea muestra de la intención del último gobernante de Bonampak por enfatizar su lazo con este antepasado, quien representa su primera conexión con Ak'e a través de su padre. Es interesante observar que el escultor de la piedra labrada haya tenido el título aj k'uhun (Jackson-Stuart, 2001; Zende, 2004), ya que el escultor del dintel 4 también tenía el mismo título. Cabe señalar que puede haber un margen de error en esta lectura, porque precisamente en esa parte la piedra se encontraba muy erosionada. 


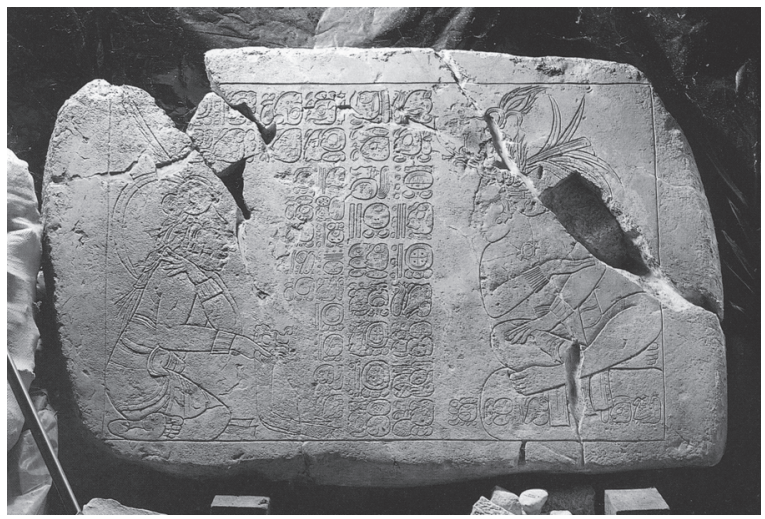

Figura 9. Piedra labrada 5

Arellano 1998: 270, lámina 5

Figura 9a. Piedra labrada 5

Dibujo de Sophia Pincemin

(Pérez Campa y Rosas Kifuri, 1987)
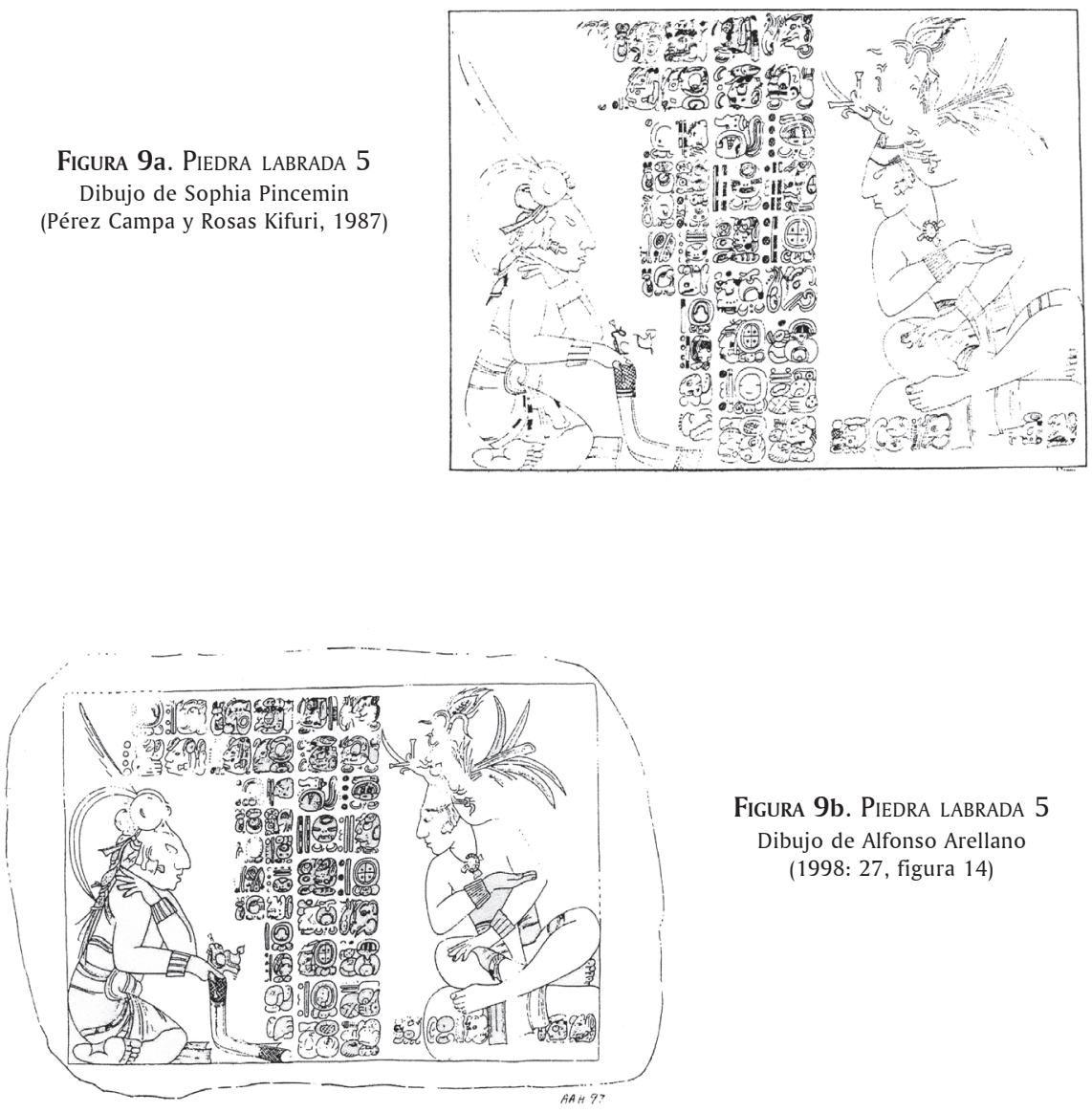

Figura 9b. Piedra labrada 5

Dibujo de Alfonso Arellano

(1998: 27, figura 14) 


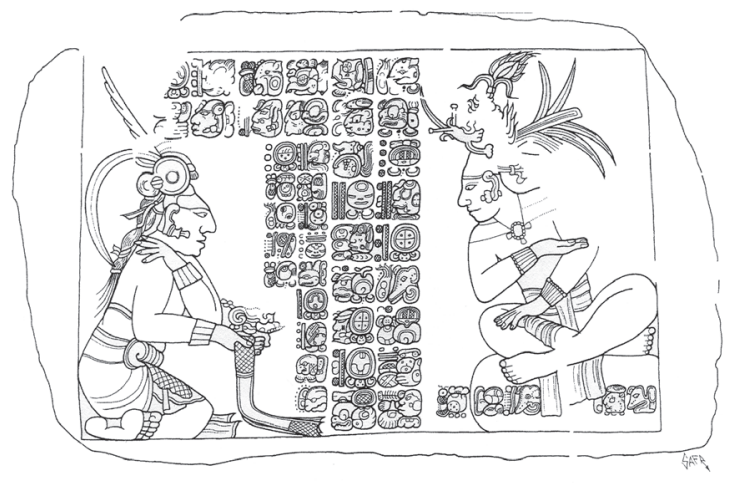

Figura 9c. Piedra labrada 5

Dibujo de Alexandr Safronov

\begin{tabular}{|c|c|}
\hline A1 3 Hix & 3 hix \\
\hline B1 $12 \mathrm{Xul}$ & $12 \mathrm{xul}$ \\
\hline A2 tu-ta-ja & tu/h/taj \\
\hline B2 NAB'-hu-k'a & nab' huk' \\
\hline A3 u-KAB'-ji-ya & u kab’jiy \\
\hline B3 AJ-SAK-la-ka-la & aj sak lakal \\
\hline A4 AJ-JUKUB' & aj jukub' \\
\hline B4 4-13-WINIK & 4 [hew] 13 winik[jiy] \\
\hline A5 tu-tu-ji-ya & tu/h]tjiy \\
\hline B5 PA'CHAN-na & pa'chan \\
\hline B6 5 Kib’ & $5 \mathrm{kib}^{\prime}$ \\
\hline B7 9 Pop & 9 рор \\
\hline B8 pe-ka-ja & pe/h]kaj \\
\hline B9 hu-? & hu? \\
\hline C1 a-na-b’i-ch'a-jo-ma & anab' ch'ajom \\
\hline D1 yi-ta-ji & yitaj \\
\hline E1 AJ-UKUL? & aj ukul? \\
\hline F1 ya-YAXUN?-B'ALAM-ma & yaxun? b'a/h/lam \\
\hline G1 PA'CHAN-AJAW & pa'chan ajaw \\
\hline H1 *7 Kib’ & 7 kib' \\
\hline I1 9 Sek & 9 sek \\
\hline H2 K’AL-ja & k'a/h/laj \\
\hline I2 HUN-na & hun \\
\hline J1 tu-B'AH & tu b'ah \\
\hline K1 WINIK-HAB'-TOK' & winik hab' tok' \\
\hline
\end{tabular}




$\begin{array}{ll}\text { J2 AJ-IXIM? } & \text { aj ixim? } \\ \text { K2 ?-u-yi } & \text { ? uy } \\ \text { L1 xu-ka-la-NAH-AJAW } & \text { Xukalnah ajaw } \\ \text { M1 u-KAB'-ji-ya } & \text { ukab'jiy } \\ \text { L2 ya-YAXUN?-B'ALAM } & \text { yaxun? b'alhjlam } \\ \text { M2 u-ti-ya } & \text { ulhtiy } \\ \text { L3 ?-TUN-ni } & \text { ? tun } \\ \text { M3 4-?-HAB'-ya } & 4 \text { ? hab'[jily } \\ \text { L4 10-WINIK-ya } & 10 \text { winik[jily } \\ \text { M4 12-K'IN-ni } & 12 \text { k'in } \\ \text { L5 CHUM-la-ji-AJAW-le } & \text { chumlajily] [ta] ajawle[l] } \\ \text { M5 8 Lamat } & \text { 8 lamat } \\ \text { L6 1 Muwan LOK'-yi-ya } & 1 \text { muwan lok'[olyiy } \\ \text { M6 u-UKU'L? } & \text { uku'l } \\ \text { L7 } 3 \text { Lamat } & 3 \text { lamat } \\ \text { M7 1 Kumk'u ?-yi } & 1 \text { kumk'u ?-Vy } \\ \text { L8 } 7 \text { K'an } & 7 \text { k'an } \\ \text { M8 12 Wo u-CH'AM } & 12 \text { wo uch'am[aw] } \\ \text { L9 nu-CHAN-pa-chi } & \text { chan pach } \\ \text { M9 ta-ma-ma-HUN?-na } & \text { ta mam hun }\end{array}$

\section{Traducción libre}

“En 3 Hix 12 Xul, Nab' Huk' fue derrotado/visitado por el de Sak Lakal y el de Jukub', 4 días y 13 veintenas después de la derrota/visita para Pa'chan, en 5 Kib' 9 Pop, Hu ? el Anab' y Derramador de Sangre y él de Ukul fueron llamados [enfrente] de Yaxun B'ahlam, el gobernante de Pa'chan. En 7 Kib' 9 Sek, Winik Hab' Tok Aj Ixim? ?-uy, el gobernante de Xukalnah, recibió "la corona" de Yaxun B'ahlam, en ? Piedra, 12 días, 10 veintenas y 4 períodos de 360 días después de su entronización, en 8 Lamat 1 Muwan, [Winikhab' Tok Aj Ixim? ?-uy] salió de Ukul?, en 3 Lamat 1 Kumk'u, [Winikhab' Tok Aj Ixim? ?-uy] regresó? para [Ukul?], en $7 \mathrm{~K}$ 'an 12 Wo, [Winikhab' Tok Aj Ixim? ?-uy] tomó el ? piel con la "corona" de su antepasado".

La piedra labrada 5 fue encontrada en 1981 entre el escombro de la escalera del Edificio 9. Mary Miller sugirió que se trataba de un tablero exterior como la piedra labrada 4 (Miller, s. f.). En la escena del monumento se aprecian dos personas sentadas dejando espacio para 45 bloques de glifos entre ellas y en el trono. La composición del monumento es similar a la de otras piedras labradas de Bonampak (1 y 4); donde también se observa un personaje sentado sobre el trono y otros frente a él. Asimismo, los tres monumentos hablan sobre la entronización de gobernantes, sus exilios y, quizás, el retorno al sitio de origen (Beliaev, 2001; Beliaev-Safronov, 2004). 
El estilo de la piedra labrada 5 corresponde al período Clásico Tardío, aunque también se puede ubicar en una etapa posterior al de la piedra labrada 4 (Miller, s. f.; Martin, en Beliaev, 2001). Se observan las siguientes ruedas calendáricas:

$\begin{array}{ll}3 \text { Hix } & 12 \text { Xul } \\ & +13.4 \\ 5 \text { Kib' } & 9 \text { Pop } \\ 7 \text { Kib' } & 9 \text { Sek } \\ & +4.10 .12 \\ \text { 8 Lamat } & 1 \text { Muwan } \\ \text { 3 Lamat } & 1 \text { Kumk'u } \\ \text { 7 K'an } & 12 \text { Wo }\end{array}$

El texto de la piedra labrada 5 menciona un cierto Yaxun B'ahlam, quien está conectado con Pa'chan o Yaxchilán, y otro topónimo que no está descifrado completamente (F1-G1 y L2). Tomando en cuenta esta condición, existen dos posibilidades de cuentas largas:

9.10.9.9.14 3 Hix 12 Xul-9.10.15.7.4 7 K'an 12 Wo (642-648)

0

9.15.15.4.10 3 Hix 12 Xul-9.16.0.15.4 7 K'an 12 Wo (746-752)

Pérez Campa y Rosas Kifuri (1987: 750-751) optaron por una cuenta larga anterior. No obstante, por su estilo y la probabilidad de que Itsamnaj B'ahlam II fuera el rey de Yaxchilán durante este período, hace que la fecha propuesta resulte una opción muy improbable.

Arellano (1998: 262) prefirió una cuenta larga entre 694-700, descartando que el glifo G1 sea una referencia Yaxchilán. En contraposición, considero que no cabe duda sobre la identificación de este glifo, planteada por primera vez por Peter Mathews (comunicación personal, 2004). El glifo en G1 es la variante de ave de uno de los signos principales de los glifos emblemas de Yaxchilán — 'el cielo partido' o pa'chan (Martin, 2004)— en combinación con el logograma AJAW.

Además, existen varios ejemplos similares en Yaxchilán, como en X1 del dintel 26, E3 del dintel 41 y glifos 45 y 163 de la escalera jeroglífica 5 (figura 10 incisos a, b, c y d). Finalmente, Nikolai Grube y Linda Schele fecharon el monumento entre 746-752, por lo que pudieron identificar al gobernante de Yaxchilán con Yaxun B'ahlam IV (Grube-Schele, 1995).

El estilo del monumento corresponde al siglo vII (Ruppert et al., 1955). Además, existen varias evidencias para aceptar una rueda calendárica en el siglo viı.

Primeramente es importante mencionar la nueva fecha dada para el dintel 3 (figuras 11, 11a y 11b). Peter Mathews propuso que la rueda calendárica más viable era 9.15.9.3.14 3 Hix 1 Kej, o sea, 740 (Mathews, 1980). No obstante, Si- 


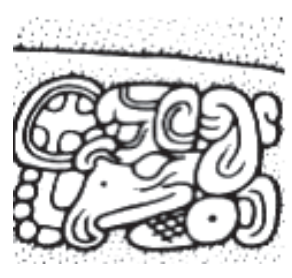

a)

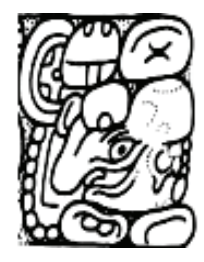

c)

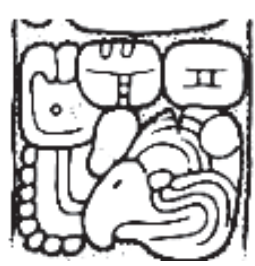

b)

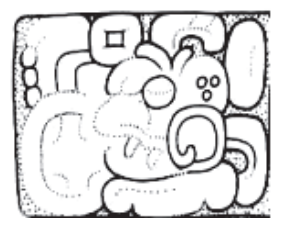

d)

Figura 10. Variante del glifo emblema de YaxchiláN

a) $\mathrm{X} 1$ del dintel $26,{ }^{10}$ b) E3 del dintel $41,{ }^{11}$

c) glifo 45 de la escalera jeroglífica $5,{ }^{12}$

d) glifo 163 de la escalera jeroglífica $5^{13}$

Dibujos de Ian Graham (1982)

mon Martin, Nikolai Grube y Christian Prager sugirieron otra fecha para la rueda calendárica 3 Hix 1 Ch'en, la cual corresponde a 9.15.17.2.13 o 748 (comunicación personal con Joel Skidmore, 2004; Martin-Miller, 2004: 80). Esta fecha podría resultar imposible porque el Hix nunca se encuentra con el número 1, pero como es una fecha correspondiente a un evento nocturno, el coeficiente del tz'olk'in se adelanta un día. Así, el 2 B'en resulta en 3 Hix (Mathews, 2001 [1979]).

10 Drawing, Glyph X1, Yaxchilan Lintel 26, from Corpus of Maya Hieroglyphic Inscriptions, vol. 3, Part 1, p. 3: 57, Yaxchilan, reproduced courtesy of the President and Fellows of Harvard College.

11 Drawing, Glyph E3, Yaxchilan Lintel 41, from Corpus of Maya Hieroglyphic Inscriptions, vol. 3, Part 2, p. 3: 91, Yaxchilan, reproduced courtesy of the President and Fellows of Harvard College.

${ }^{12}$ Drawing, Glyph 45, Yaxchilan Hieroglyphic Stairway 5, from Corpus of Maya Hieroglyphic Inscriptions, vol. 3, Part 3, p. 3: 179, Yaxchilan, reproduced courtesy of the President and Fellows of Harvard College.

${ }^{13}$ Drawing, Glyph 163, Yaxchilan Hieroglyphic Stairway 5, from Corpus of Maya Hieroglyphic Inscriptions, vol. 3, Part 3, p. 3: 181, Yaxchilan, reproduced courtesy of the President and Fellows of Harvard College 
Entonces, del dintel 3 se sabe que el rey de Xukalnah y Ak'e, en 748, fue Aj Sak Telech, el padre de Yajaw Chan Muwan II (Mathews, 1980; Martin-Miller, 2004: 80).

Si tenemos en cuenta este hecho, resulta difícil interpretar que cierto Winikhab' Tok' hubiera sido el rey entre 747 y 752 . Dicha evidencia indica que la fecha de la piedra labrada 5 debe estar entre 642-648.

Además, la entronización del gobernante de Xukalnah se llevó a cabo en un lugar no identificado, recibiendo su "corona" de Yaxun B'ahlam. Con el fechamiento de 746-752, correspondiendo a la entronización en 9.15.15.16.16, Yaxun B'ahlam IV no sería el rey de Yaxchilán sino, posiblemente, fuera Sak Jukub' Yopat B'ahlam, mencionado en el panel 3 de Piedras Negras (Schele-Grube, 1995: 111; Martin-Grube, 2000: 127).

Esas evidencias indican que la piedra labrada 5 conmemora eventos entre 9.10.9.9.14-9.10.15.7.4 (642-648), y durante ese tiempo el rey de Yaxchilán era Aj Wak Tun Yaxun B'ahlam III (629-681?). Según esta interpretación, el rey de Xukalnah entre 643 y 648 fue Winikhab' Tok, que probablemente fue un subordinado del gobernante de Yaxchilán.

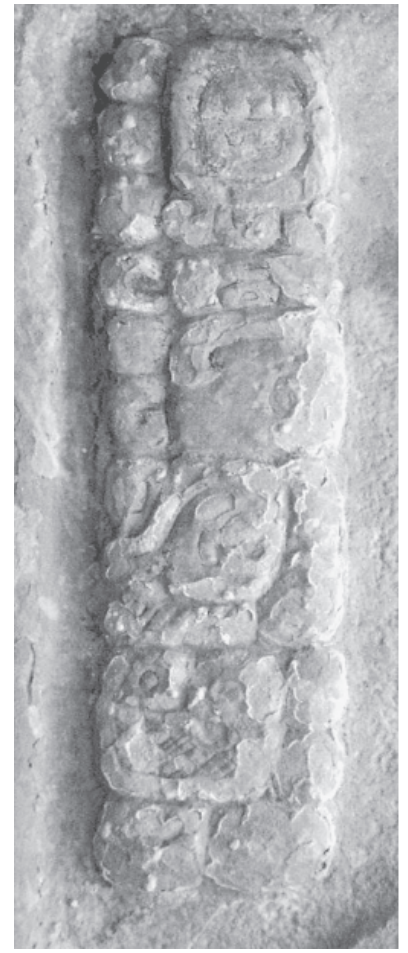

Figura 11. Dintel 3 de Bonampak Foto de Joel Skidmore 


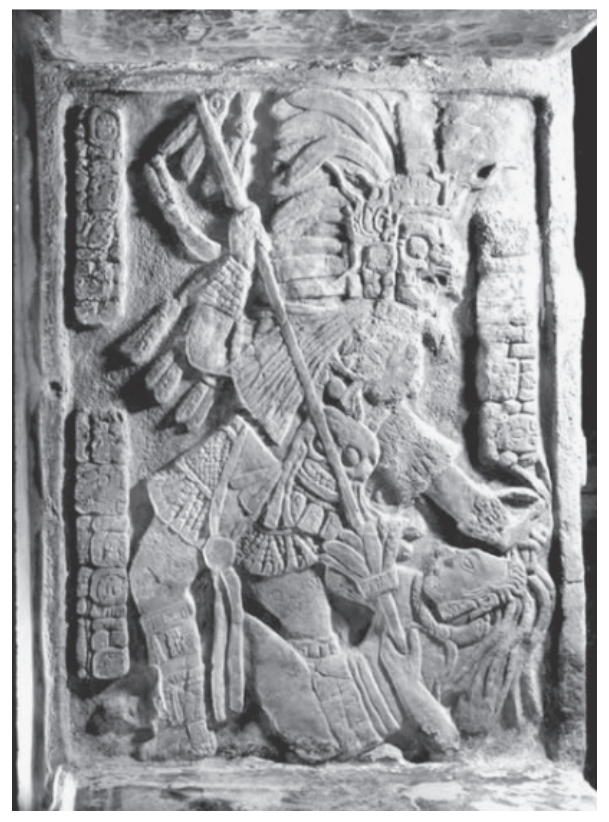

Figura 11a. Dintel 3 de Bonampak Foto de Joel Skidmore

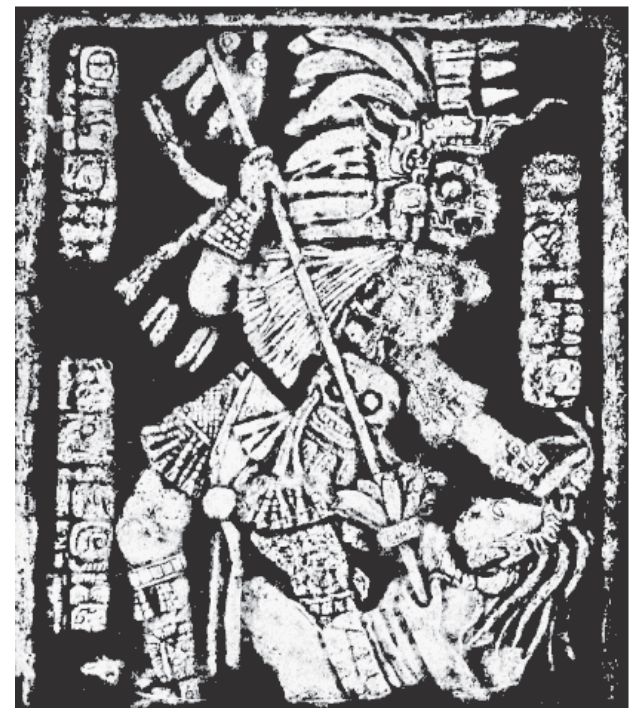

Figura 11b. Dintel 3 de Bonampak

Calca hecha por Merle Greene Robertson 


\section{Consideraciones finales}

Las piedras labradas 2, 4 y 5 son los monumentos más tempranos del sitio arqueológico de Bonampak y conmemoran, en detalle, la dificultad de gobernar una ciudad maya en la época Clásica. Las piedras labradas 4 y 5 relatan el exilio de los gobernantes con el título Xukalnah Ajaw, y mencionan otros dos de Yaxchilán, quienes los ayudaban activamente. Es muy probable, incluso, que los gobernantes de Yaxchilán fueran señores supremos mientras que los reyes de Bonampak eran sus subordinados.

Según mi reconstrucción, la piedra labrada 2 fue dedicada por Yajaw Chan Muwan I alrededor de 605, y junto con el dintel 4 de Bonampak son los únicos monumentos de este importante rey. Yajaw Chan Muwan I se entronizó en 9.8.6.13.17 y murió en 9.8.12.2.2. Este presonaje fue hijo del gobernante de Ak'e, un sitio antes identificado con Bonampak mismo (Mathews, 1980), y de una señora con rango sajal de un sitio desconocido, tal vez llamado K'an Tok Luk.

La piedra labrada 4 fue dedicada alrededor de 9.9.1.7.1 y conmemora los eventos de la vida de Yajaw Chan Muwan I, la muerte de Ix K'an Tok Luk y, sobre todo, menciona al siguiente gobernante de Xukalnah: Aj ? Nal, quien se entronizó en 9.8.12.2.9. Pronto hubo problemas en su reinado, porque otro personaje, Aj ? Chan Tok'?, fue el gobernante con el título Xukalnah Ajaw. Aj ? Nal fue exiliado a Yaxchilán y para poder recuperar su reinado tal vez necesitó la ayuda de Itsamnaj B’ahlam II, un gobernante de Yaxchilán sin mención en las inscripciones de su ciudad.

La piedra labrada 5 fue dedicada alrededor de 9.10.15.7.4 y conmemora dos eventos distintos y, me parece, que los conflictos/visitas mencionados al comienzo del texto ante la presencia de Yaxun B’ahlam III de Yaxchilán fueron importantes en el reconocimiento de la entronización de Winikhab' Tok'. El exilio y regreso de Winikhab' Tok' a Bonampak muestran, de nuevo, los problemas y, tal vez, conflictos entre diferentes facciones de una familia real, que implicaba el gobierno de una ciudad maya durante la época Clásica.

El siguiente monumento de Bonampak es la piedra labrada 1 que se fecha 9.13.0.0.0 (Mathews 1980) y menciona la entronización de Aj Ixim? K’ey en 9.12.11.6.9. Aunque existen varias brechas en la secuencia de los gobernantes de Bonampak en el siglo vil (figura 12), ya no hay tanto problema para explicar la presencia de varios gobernantes durante el mismo tiempo en el siglo vilI.

El único glifo emblema mencionado en las piedras labradas 1, 2, 4, 5 y el dintel 4 es Xukalnah Ajaw, salvo la mención de Ak'e como el glifo emblema del padre de Yajaw Chan Muwan I. Sin embargo, la entronización de Yajaw Chan Muwan I ocurre en un lugar cuyo nombre se escribe con el silabograma u y una cabeza de ave no descifrada (PL 4, B3; figura 13 inciso a). Tal vez el mismo topónimo esté mencionado en D8 de la piedra labrada 5. Winikhab' Tok' salió del mismo lugar, de nuevo escrito con el silabograma u y la cabeza de ave en M6 de la piedra labrada 5 (figura 13 inciso b). Sin el silabograma u, pero con 


\begin{tabular}{|c|c|c|}
\hline $\begin{array}{l}\text { Ch'ak Kab'an Yajaw Chan } \\
\text { Muwan I }\end{array}$ & 9.8.6.13.17-9.8.12.2.2. & $\begin{array}{l}\text { dintel } 4 \text {, piedra labrada } 2 \text {, } \\
\text { mención en piedra } \\
\text { labrada } 4\end{array}$ \\
\hline Aj ? Nal? & $9.8 .12 .2 .9-9.8 .17 .15 .0<$ & piedra labrada 4 \\
\hline Aj ? Chan Tok' & 9.8.16.14.10?-9.8.17.15.0 & $\begin{array}{l}\text { mención en la piedra } \\
\text { labrada } 4\end{array}$ \\
\hline Aj Ixim? Winikhab’ Tok' ?-uy & $9.10 .10 .8 .16-9.10 .15 .7 .4<$ & piedra labrada 5 \\
\hline Aj Ixim? K'ey & $9.12 .11 .6 .9-9.13 .0 .0 .0<$ & piedra labrada 1 \\
\hline Yajawte' K'inich Chak Chij & 9.15.5.0.0?-9.15.0.13.6? & $\begin{array}{l}\text { menciones en los paneles } \\
\text { de Zurich y de El Cedro- } \\
\text { Nuevo Jalisco }\end{array}$ \\
\hline ? B'ahlam & 9.15.0.13.6?-9.15.15.0.0< & $\begin{array}{l}\text { paneles de El Cedro- } \\
\text { Nuevo Jalisco, mención en } \\
\text { dintel } 3 \text { de Bonampak }\end{array}$ \\
\hline Aj Sak Telech & $>9.15 \cdot 17.2 .3-9 \cdot 17 \cdot 5 \cdot 8.9 ?$ & $\begin{array}{l}\text { dintel de Kuná-Lakanha, } \\
\text { menciones en dintel } 3 \text {, } \\
\text { estelas } 1 \text { y } 2 \text { de Bo- } \\
\text { nampak }\end{array}$ \\
\hline Yajaw Chan Muwan II & 9.17.5.8.9-9.18.1.2.0< & $\begin{array}{l}\text { estelas } 1,2,3 \text { y } 5 \text {, } \\
\text { dinteles } 1,2 \text { y } 3 \text { de } \\
\text { Bonampak, murales del } \\
\text { Edificio } 1 \text { de Bonampak }\end{array}$ \\
\hline
\end{tabular}

Figura 12. Reyes, fechas y monumentos en Bonampak

el afijo agentivo AJ, la cabeza de ave designa un personaje que fue invocado en frente de Yaxun B'ahlam III, como se conmemora en el mismo monumento (PL 5, E1; figura 13 inciso c). En la piedra labrada 1 se encuentra la misma combinación de glifos después del verbo ?-yi (tal vez 'regresar', Beliaev, 2001) y la expresión $t u k a b$ ' ch'en que normalmente señala que el siguiente bloque de glifo contiene un topónimo (Stuart-Houston, 1994; figura 13 inciso d). Aj Sak Telech, el padre de Yajaw Chan Muwan II también lleva el mismo topónimo antes de los glifos emblemas de Xukalnah y Ak'e en la estela 2 de Bonampak (E5; figura 13 inciso e), ahora escrito con el afijo agentivo AJ, la cabeza de ave y el silabograma la. Finalmente, en el dintel 1 de Bonampak (B4; figura 13 inciso f), Yajaw Chan Muwan II tiene el mismo topónimo escrito como u y la cabeza de ave. 


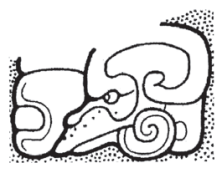

a)

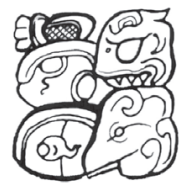

d)

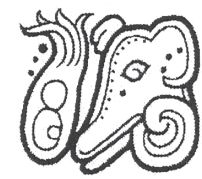

b)

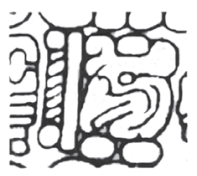

e)

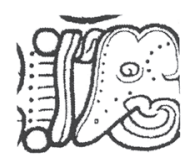

c)

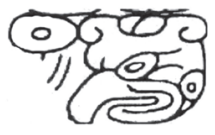

f)

FigURA 13. UKUL? a) u-UK? (piedra labrada 4, B3)

b) u-UK? (piedra labrada 5, M6)

c) AJ-UK? (piedra labrada $5, \mathrm{E}$ )

d) tu-KAB' tu CH'EN u-UK? (piedra labrada 1, D2)

e) AJ-UK?-la (estela 2, F5)

f) u-UK? (dintel 1, B4).

Incisos a, b y c, dibujos de Alexandr Safronov.

Incisos d, e y f, dibujos de Peter Mathews (1980, figuras 2, 9 y 5)

Dmitri Beliaev (2001) había sugerido que la cabeza de ave se leyera li, pero más tarde, con Alexandr Safronov, ha sugerido una lectura UK o UKUL (BeliaevSafronov, 2004). Esta última lectura toma en cuenta que el topónimo comienza con el fonema $u$ y termina en $-V l$, y los autores sugieren que es igual que el topónimo de uno de los cautivos de Yaxun B'ahlam IV, un cierto Aj Uk o Aj Ukul. Aunque la lectura es probable, sería necesario encontrar una substitución directa para poder asegurarlo.

No obstante, apoyándome en su mención en las inscripciones de Bonampak, estoy de acuerdo con Dmitri Beliaev y Alexandr Safronov (2004) de que Uk o Ukul sea el nombre de este sitio o una parte de él, tal vez la Acrópolis. Uk o Ukul con los sitios Sak Lakal, B'ub'ul Ha' (Ojos de Agua?), Jukub' y Patal? tenían en común que sus gobernantes llevaron el título Xukalnah Ajaw. Es difícil interpretar esta situación, pero opino que Xukalnah debe ser el nombre de un sitio desconocido y, además, los gobernantes de este sitio llevaron el título Xukalnah Ajaw o 'Señor de Xukalnah'. Sin embargo, desde finales del siglo vı hay evidencias de que varias dinastías, en sitios distintos como Bonampak y Ojos de Agua, 
se separaron de la familia de Xukalnah, y fundaron sus propias entidades políticas en varios sitios como los recién mencionados.

De acuerdo con esta interpretación, Ak'e es un sitio desconocido también, aunque su historia está conectada con la de Uk o Ukul, al menos desde el tiempo de Yajaw Chan Muwan I, y, más estrechamente, durante la época de ?B'ahlam, Aj Sak Telech y Yajaw Chan Muwan II, más o menos en la segunda mitad del siglo viII.

La separación de varias ramas de una familia real y la fundación de nuevos sitios tal vez fue un fenómeno que ocurrió en los comienzos del Clásico Temprano. Varias ciudades de la región del Usumacinta mencionan lazos con los sitios del Petén central en inscripciones retrospectivas. Quizás investigaciones arqueológicas en el futuro aclaren más este fenómeno.

\section{Agradecimientos}

Durante la investigación, el autor recibió sugerencias y ayuda de varias personas, como Peter Mathews, Maricela Ayala, Dmitri Beliaev, Alexandr Davletshine, Joel Skidmore, Marc Zender, y Claudia García Solís, a ésta última mi agradecimiento por su apoyo en la revisión de la redacción. Sin embargo, las ideas y errores en el artículo son responsabilidad del autor. Esta investigación fue posible por una beca de la Universidad La Trobe, Melbourne, Australia.

\section{BIBLIOGRAFÍA}

Arellano Hernández, Alfonso

1998 "Diálogo con los abuelos”, La pintura mural prehispánica en México II, Area Maya, Bonampak, Tomo II, Estudios, pp. 255-297, Staines Cicero (ed.). México: UNAM, Instituto de Investigaciones Estéticas.

Beliaev, Dmitri

T550 as a Verb for "Return" [2001], manuscrito inédito.

Beliaev, Dmitri y Alexandr Safronov

2004 Ak'e и Шукальнах: история и политическая география государств майя Верхней Үсумасинты (www.mesoamerica.ru/indians/maya/ake.html).

Bíró, Péter

2007 "Una propuesta para el análisis de los textos mayas de la época Clásica”, Estudios Mesoamericanos, 7, pp. 15-28, México: UNAM, FFyL, IIFL.

Colas, Pierre-Robert

2003 "K'inich and king: naming self and person among Classic Maya rulers", Ancient Mesoamerica, 14: 269-283. Cambridge: Cambridge University Press. 
Graham, Ian

1982 Yaxchilan, Corpus of Maya Hieroglyphic Inscriptions, vol. 3 (3). Cambridge: Peabody Museum or Archaeology and Ethnology, Harvard University.

Grube, Nikolai

1996 "Palenque in the Maya world", Eighth Palenque Round Table, 1994, pp. 1-13, Merle Greene Robertson y Virginia Fields (eds.). San Francisco: San Francisco Pre-Columbian Art Research Institute.

2001 "Los nombres de los gobernantes mayas", Arqueología Mexicana 9(50): 72-77. México: Raíces.

s. f. "Commentary to vase K8777", Maya Vase Database (http://www.mayavase. com/ 8777/com8777.html).

Grube, Nikolai y Linda Schele

1995 The Last Two Hundred Years of Maya History, Workbook for the XIXth Maya Hieroglyphic Workshop at Texas. Austin: Department of Art History, University of Texas.

Houston, Stephen D., David Stuart y John S. Robertson

1998 "Disharmony in maya hieroglyphic writing: linguistic change and continuity in Classic society", Anatomía de una civilización: Aproximaciones interdisciplinarias a la cultura maya, pp. 275-26, Andrés Ciudad Ruiz, Yolanda Fernández, José Miguel García Campillo, Ma. Josefa Iglesias Ponce de León, Alfonso Lacadena García-Gallo y Luis T. Sáenz Castro (eds.). Madrid: Sociedad Española de Estudios Mayas (Publicaciones de la SEEM, 4).

Jackson, Sarah y David Stuart

2001 "The Aj K'uhun title: deciphering a Classic Maya term of rank", Ancient Mesoamerica 12: 217-228. Cambridge: Cambridge University Press.

Lacadena, Alfonso

1995 "Las escrituras logo silábicas: el caso maya", Estudios de Historia Social y Económica de América, 12: 601-607. España: Universidad de Alcalá de Hénares.

Lacadena, Alfonso y Soren Wichmann

2004 "On the representation of the glottal stop in Maya writing", The Linguistics of Maya Writing, pp. 100-164, Soren Wichmann (ed.). Salt Lake City: The University of Utah Press.

MacLeod, Barbara

2004 "A word in a grain of sand: transitive perfect verbs in the Classic Maya script", The Linguistics of Maya Writing, pp. 291-326, Soren Wichmann (ed.). Salt Lake City: The University of Utah Press. 
Martin, Simon

2004 "A broken sky: the ancient name of Yaxchilan as Pa' Chan", The PARI Journal 5(1): 1-7. San Francisco: Precolumbian Art Research Institute.

Martin, Simon y Nikolai Grube

2000 Chronicle of Maya King and Queens: Deciphering of the Ancient Dynasties of the Ancient Maya. Londres: Thames and Hudson.

Mathews, Peter

1980 "Notes on the dynastic sequence of Bonampak, part 1", Third Palenque Round Table, Part 2: 60-73, Merle Greene Robertson (ed.). Austin: University of Texas Press.

1988 “The sculptures of Yaxchilan”. Tesis doctoral. New Haven: Yale University.

2001 "The inscription on the back of Stela 8, Dos Pilas, Guatemala", The Decipher[1979] ment of Ancient Maya Writing, pp. 394-418, Stephen D. Houston, David Stuart y Osvaldo Chinchilla Mazariego (eds.). Norman: University of Oklahoma Press.

Miller, Mary

s. f. Notes on the Dynastic Sequence of Bonampak, Part 2, manuscrito inédito.

Miller, Mary y Simon Martin (eds.)

2004 Courtly Art of the Ancient Maya. San Francisco: Fine Arts Museum of San Francisco, Thames and Hudson.

Mora-Marín, David

2003 "Affixation convantionalization: an explanation of regularly dysharmonic spellings in Mayan writing" (http://www.unc.edu/ davidmm/AffixationConvention. pdf).

Nahm, Werner

1997 "Hieroglyphic stairway 1 at Yaxchilan”, Mexicon 19 (1): 65-69. Alemania: Verlag Anton Saurwein.

Pérez Campa, Mario y Mauricio Rosas Kifuri

1987 "Dos nuevas piedras labradas de Bonampak", Memorias del Primer Coloquio Internacional de Mayistas, pp. 749-773. México: UNAM, Centro de Estudios Mayas.

Ruppert, Karl et al.

1955 Bonampak, Chiapas, Mexico. Washington: Carnegie Institution of Washington (Publication, 602).

Stuart, David y Stephen D. Houston

1994 Classic Maya Place Names. Washington: Dumbarton Oaks (Studies in Precolumbian Art and Archaeology, 33). 
Tate, Carolyn Elaine

1992 Yaxchilan: the Design of a Maya Ceremonial City. Austin: University of Texas Press.

Tovalían Ahumada, Alejandro y Víctor Manuel Ortiz Villarreal

2002 "La cripta funeraria asociada al Edificio 4 de Bonampak, Chiapas", Lakamha' 5: 4-10. Palenque: Museo y Zona Arqueológica de Palenque.

Wagner, Elizabeth

2003 "The female title prefix", Wayeb Notes (www.wayeb.org/notes/wayeb_notes 0005.pdf).

2004 The Inscription and Iconography of Bonampak, Sculptured Stone 4: Evidence for a yet Unknown Early Ruler of Yaxchilan, manuscrito inédito.

Wald, Robert

2000 "Temporal deixis in colonial Chontal and Maya hieroglyphic narrative", Written Language and Literacy 3(1):123-153. The Netherlands: University of Nijmegen.

2004 "Telling time in Classic-Ch'olan and Acalan-Chontal narrative: the linguistic basis of some temporal discourse patterns in Maya hieroglyphic and AcalanChontal texts", The Linguistics of Maya Writing, pp. 211-258, Soren Wichmann (ed.). Salt Lake City: The University of Utah Press.

Zender, Marc

1999 "Diacritical marks and underspelling in the Classic Maya script: implications for decipherment”. Tesis de maestría. Calgary: Departamento de Arqueología, Universidad de Calgary.

2004 "A study of Classic Maya priesthood". Tesis doctoral. Calgary: Departamento de Arqueología, Universidad de Calgary. 